\title{
Sinorhizobium meliloti 1021 Loss-of-Function Deletion Mutation in chvl and Its Phenotypic Characteristics
}

\author{
Chunxia Wang, ${ }^{1}$ Jocelyn Kemp, ${ }^{1}$ Isabel O. Da Fonseca, ${ }^{1}$ Raymie C. Equi, ${ }^{1}$ Xiaoyan Sheng, ${ }^{1}$ \\ Trevor C. Charles, ${ }^{2}$ and Bruno W. S. Sobral ${ }^{1}$ \\ ${ }^{1}$ Virginia Bioinformatics Institute, Virginia Polytechnic Institute and State University, Blacksburg 24061, U.S.A.; ${ }^{2}$ Department \\ of Biology, University of Waterloo, Waterloo, ON, N2L 3G1, Canada
}

Submitted 14 August 2009. Accepted 15 October 2009.

\begin{abstract}
Bacterial two-component regulatory systems (TCS) are common components of complex regulatory networks and cascades. In Sinorhizobium meliloti, the TCS ExoS/ChvI controls exopolysaccharide succinoglycan production and flagellum biosynthesis. Although this system plays a crucial role in establishing the symbiosis between $S$. meliloti and its host plant, it is not well characterized. Attempts to generate complete loss-of-function mutations in either exoS or chvI in S. meliloti have been unsuccessful; thus, it was previously suggested that exoS or chvI are essential genes for bacterial cell growth. We constructed a chvI mutant by completely deleting the open reading frame encoding this gene. The mutant strain failed to grow on complex medium, exhibited lower tolerance to acidic condition, produced significantly less poly-3-hydroxybutyrate than the wild type, was hypermotile, and exhibited an altered lipopolysaccharide profile. In addition, this mutant was defective in symbiosis with Medicago truncatula and M. sativa (alfalfa), although it induced root hair deformation as efficiently as the wild type. Together, our results demonstrate that ChvI is intimately involved in regulatory networks involving the cell envelope and metabolism; however, its precise role within the regulatory network remains to be determined.
\end{abstract}

Bacteria have developed signaling systems for eliciting a variety of adaptive responses to their environments. Two-component regulatory systems (TCS) are one of the most important mechanisms for these adaptive responses. They consist of two protein components: a sensor that monitors some environmental parameters and a response regulator that mediates a change in gene expression in response to sensor signals ( $\mathrm{Li}$ et al. 2002). They are found in the majority of bacteria, and they control housekeeping functions as well as regulate genes important for pathogenesis, stress, and adherence (Azcarate-Peril et al. 2005; Nishino et al. 2005). Recently, more than 4,000 putative TCS have been identified in 145 sequenced bacterial genomes (Ulrich et al. 2005). In Sinorhizobium meliloti, at least 31 putative histidine kinase sensors (based on RhizoBase) and 30 putative response regulators have been found during analyses of the S. meliloti 1021 genome. The functions of many of these putative systems remain unknown.

The gram-negative $\alpha$-proteobacterium $S$. meliloti, found in soil, enters into a nitrogen-fixing symbiosis within the roots of leguminous plants such as Medicago truncatula and $M$. sativa (alfalfa). A successful symbiosis involves a complex series of

Corresponding author: Chunxia Wang; Telephone: +1 540231 1958; Fax: +1 5402312606 E-mail: cwang@vbi.vt.edu interactions and communication events between the host and bacteria (Long 1989, 2001; Brewin 1991; van Rhijn and Vanderleyden 1995; Jones et al. 2007). Initially, plants release flavonoids that trigger the rhizobial bacteria to produce lipochitin oligosaccharide compounds called Nod factors (Perret et al. 2000). The Nod factors then induce root hair curling and root nodule development. In addition to the Nod factor, $S$. meliloti produces exopolysaccharides (EPS) called succinoglycan (EPSI) and galactoglucan (EPSII) (Leigh et al. 1985; Finan et al. 1986) that is required for the formation of infection threads inside root hairs (Cheng and Walker 1998a). $S$. meliloti cells invade the developing root nodule via a cell division process within the infection thread. Once released from the infection threads, the bacterial cells differentiate into bacteroids and convert atmospheric nitrogen to ammonia (Schultze and Kondorosi 1998; Oke and Long 1999; Pellock et al. 2000).

In $S$. meliloti, the exoS and $c h v I$ genes constitute a TCS that plays a role in controlling EPSI production (Cheng and Walker 1998b). S. meliloti Rm1021 mutants that fail to synthesize EPSI form empty nodules that are devoid of bacteria or bacteroids (Leigh et al. 1985; Pellock et al. 2000) and are unable to establish a symbiosis because they are defective in initiating the production of infection threads and in invading the plant (Mendrygal and Gonzalez 2000; Jones et al. 2008). S. meliloti exo $S$ has been studied extensively. Previous studies suggest that exoS and chvI are essential genes for growth under standard laboratory conditions, because attempts to generate complete loss-of-function mutations in either exoS or chvI in $S$. meliloti have been unsuccessful (Osteras et al. 1995; Cheng and Walker 1998b; Wells et al. 2007). Although most insertion mutations in exoS and $c h v I$ were believed to be lethal (Osteras et al. 1995), a Tn5 insertion $57 \mathrm{bp}$ from the $\mathrm{N}$ terminus within the open reading frame (ORF) (named exoS96::Tn5 mutation) results in the production of a constitutively active ExoS sensor kinase which upregulates EPSI levels. This results in the overproduction of EPSI (Doherty et al. 1988), presumably via the cognate response regulator ChvI (Cheng and Walker 1998b), and modulates expression of other exo genes (Doherty et al. 1988; Wells and Long 2002; Cheng and Yao 2004). In addition to the regulation of EPSI, the ExoS/ChvI system in S. meliloti is also involved in regulating flagellum biosynthesis (Yao et al. 2004). A recent study shows that ExoS/ChvI may interact with ExoR and control the expression of multiple transcriptional regulators (Wells et al. 2007). However, exoS mutants that have been studied to date are, in fact, constitutively active rather than null mutants (Cheng and Walker 1998b). No lossof-function mutations in either exoS or chvI have been reported in S. meliloti thus far. 
Although the outcome is different, common mechanisms exist in pathogenic and symbiotic bacteria to support successful colonization of the host cells (Tsolis 2002). Studies of the ExoS/ ChvI homologues in other $\alpha$-proteobacteria have revealed that they regulate the expression profile of proteins associated with the bacterial cell envelope, and are required for persistence and virulence within host cells (Wells et al. 2007). For example, the ChvG/ChvI system is required for vir gene expression, virulence, and growth under acid conditions in Agrobacterium tumefaciens (Charles and Nester 1993; Li et al. 2002; Yuan et al. 2008). The homologue BvrS/BvrR is required for intracellular survival and controls the cell invasion and virulence of Brucella abortus in animal cells (Sola-Landa et al. 1998). Previous evidence also indicates that $\mathrm{BvrS} / \mathrm{BvrR}$ controls the expression of several outer membrane proteins and is involved in modulating the properties of bacterial lipopolysaccharide (LPS) (Guzman-Verri et al. 2002; Manterola et al. 2005; Lamontagne et al. 2007).

Herein, we deleted the ORF of the $S$. meliloti Rm1021 chvI gene. Further, it is shown that the chvI mutant fails to grow in complex media or acidic media. The mutant exhibits symbiotic deficiency but is able to induce root hair deformation. In addition, loss of ChvI function disrupts intracellular polymer accumulation and affects cell motility. These findings provide additional insights into the role of ExoS/ChvI during the early stages of symbiosis.

\section{RESULTS}

Construction and phenotype of $\operatorname{chvI}$ mutant strain SB114.

A complete loss-of-function mutation of $c h v I$ in $S$. meliloti Rm1021 was generated by crossover polymerase chain reaction (PCR) (Sukdeo and Charles 2003) using the suicide vector pK19mobsacB (Schafer et al. 1994). The presence of the dele-
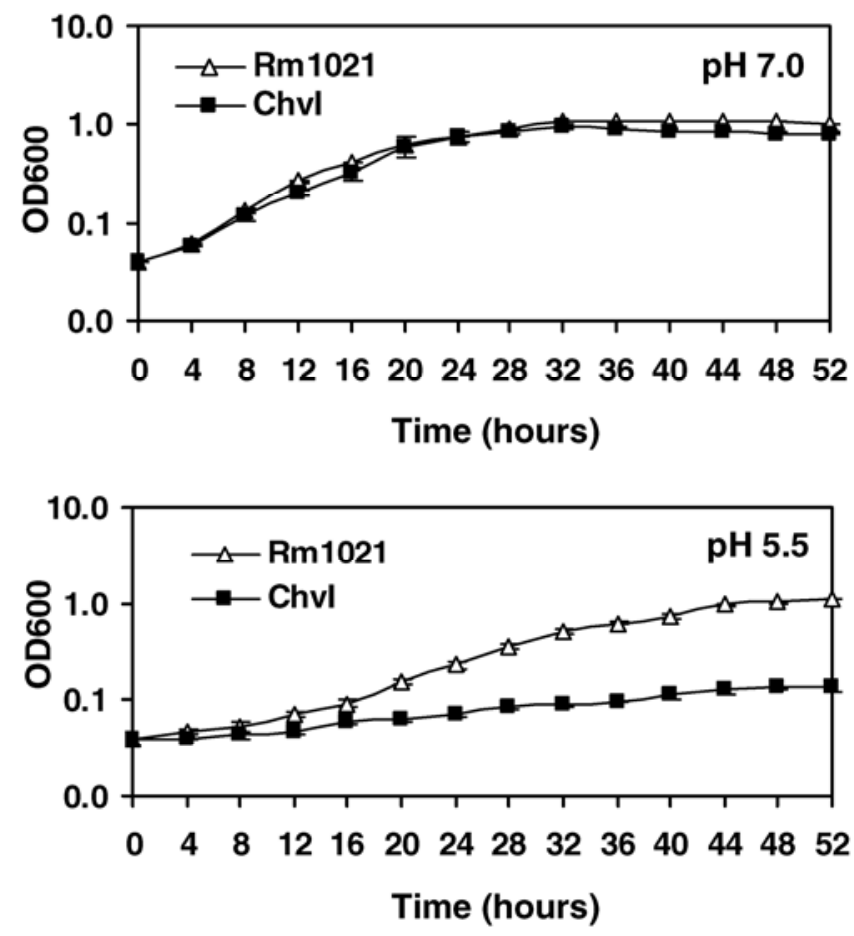

Fig. 1. Growth curve of Sinorhizobium meliloti strains in M9+ succinate (M9 + suc.) minimal medium. Log-phase cultures of the wild-type Rm1021 and the $c h v I$ mutant from M9+suc. were inoculated into fresh M9+ suc. medium (top, $\mathrm{pH} 7.0$; bottom, $\mathrm{pH} 5.5$ ) with a starting optical density at $600 \mathrm{~nm}\left(\mathrm{OD}_{600}\right)$ of 0.04 . The cells were grown at $30^{\circ} \mathrm{C}$ with shaking. The $\mathrm{OD}_{600}$ values are the means of three independent experiments. tion was confirmed by PCR and DNA sequencing. We were unable to generate the knockout $c h v I$ mutant on tryptone yeast (TY) medium but we generated such a mutant by using M9 + succinate (M9 + suc.) medium. This mutant grew well on YMB (Tombolini et al. 1995) but was unable to grow in media containing tryptone. For complementation analysis, plasmid pCW681 was constructed harboring the complete chvI ORF cloned in the broad-host-range vector pBBR1MCS-5 (Kovach et al. 1995). All phenotypic defects such as growth in different media, motility, polymer accumulation, and symbiotic properties in the chvI mutant, could be complemented by pCW681.

\section{The $c h v I$ mutant is inhibited by an acidic environment.}

TCS $c h v G-c h v I$ is critical for virulence and is induced under acid conditions in Agrobacterium spp. (Yuan et al. 2008), and the $S$. meliloti exoS and chvI genes are highly similar to the $A$. tumefaciens chvG and $c h v I$ genes, respectively. Therefore, we determined whether the $S$. meliloti chvI deletion mutant was acid sensitive by monitoring the growth behavior of both the chvI mutant and the wild-type strain Rm1021 (Fig. 1). The chvI mutant grew normally compared with the wild-type Rm1021 at $\mathrm{pH} 7.0$ but more poorly than $\mathrm{Rm} 1021$ at $\mathrm{pH}$ 5.5. Also, in comparison with the growth rates of wild-type strain Rm1021 at acidic and neutral conditions, the growth rate was slightly lower at $\mathrm{pH} 5.5$ than at $\mathrm{pH} 7.0$; however, it still exhibited steady-state exponential growth. Thus, the acidic ( $\mathrm{pH} 5.5)$ growth conditions conferred mild acid stress on the cells.

\section{Loss of ChvI function disrupts LPS production} and EPS and poly-3-hydroxybutyrate accumulation.

To test whether ExoS/ChvI is involved in modulating the properties of bacterial LPS, as is the case for BvrS/BvrR in $B$. abortus, we extracted and analyzed the LPS from the wild type and the $c h v I$ mutant in S. meliloti. Resolution of LPS molecules by polyacrylamide gel electrophoresis followed by silver staining revealed striking differences between LPS from the wild-type Rm1021 and that of the chvI mutant. The smooth LPS band (higher molecular weight) was not detected in the mutant; however this phenotype was complemented by the plasmid-carried chvI gene (Fig. 2). To determine the growth behavior of strains and their ability to synthesize and accumu-
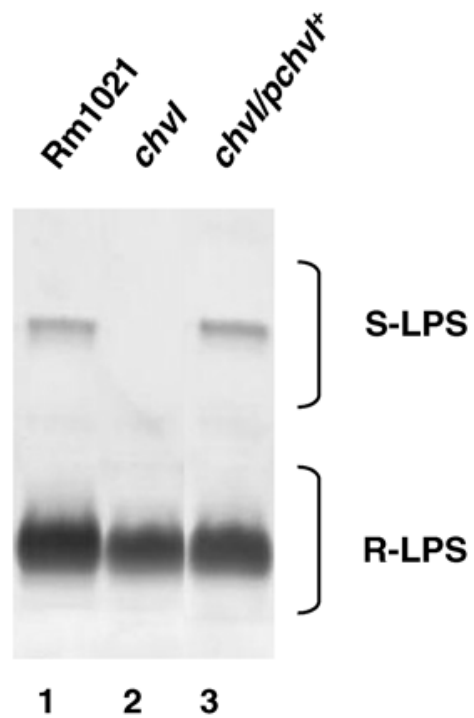

Fig. 2. Lipopolysaccharide profiles in the wild-type strain Rm1021 and the chvI mutant after resolution by sodium dodecyl sulfate polyacrylamide gel electrophoresis and silver staining. Lane 1, the wild-type strain Rm1021; lane 2, the chvI mutant strain; lane 3, the complemented strain SB1201. Sand R-LPS = smooth and rough lipopolysaccharide, respectively. 
late EPS and poly-3-hydroxybutyrate (PHB), the chvI mutant and wild-type strain were cultivated in liquid YMB medium. The wild-type strain and the chvI mutant showed similar growth kinetics (Table 1); however, EPS was not detected in the $c h v I$ mutant. This observation is consistent with an essential role for ChvI in the regulation of EPS synthesis. Surprisingly, we found that the $c h v I$ mutation resulted in significantly less PHB production than the wild-type strain (Table 1).

Carbon source utilization patterns were also determined on M9 agar with either succinate, fructose, sucrose, glucose, acetoacetate, or pyruvate as the sole carbon source. All strains had similar growth behaviors on succinate, fructose, sucrose, and glucose. However, the $c h v I$ mutant grew slightly more slowly, forming smaller colonies after 4 days of growth than those of the wild type on acetoacetate or pyruvate (data not shown).

\section{The $c h v I$ mutation affects apparent cell motility.}

Previous studies showed that the exoS96 mutant is severely impaired in expression of flagellar biosynthesis genes and cell motility in comparison with the wild type (Yao et al. 2004). To examine whether the $c h v I$ mutation has a phenotype similar to the exoS96 mutation, we assayed the strains for motility and the presence or absence of flagella. The wild-type strain Rm1021, its EPS-deficient exoY mutant Rm7210 (Leigh et al. 1985), and its nonmotile, flagellaless flip mutant RmG910 (Finan et al. 1995) were similarly tested on swim plates as controls. Strains capable of motility showed an increased diameter in colony size after 4 days compared with the strain incapable of swimming. All strains tested were capably motile in the swim plates, except for the nonmotile control, flip mutant RmG910 (Fig. 3A). Both the wild-type strain Rm1021 and the exoY mutant strain Rm7210 formed small, tightly packed colonies, and the chvI mutant formed large, diffuse colonies (Fig. 3A), suggesting that large colonies of the chvI mutant do not result from EPS production. To determine whether the apparent increase of motility of $c h v I$ was related to the presence of flagella, wild-type and chvI mutant cells were stained for flagella and examined in detail by using transmission electron microscopy (TEM). Both wild-type Rm1021 and chvI mutant cells grown in the swim plates containing minimal media (M9 + suc.) were flagellated (Fig. 3B and C). These findings raised the possibility that ChvI may control cell motility genes, directly or through other regulators.

\section{Loss of ChvI function causes symbiotic defects but doesn't affect ability to induce root hair deformation.}

To examine the symbiotic phenotype of the chvI mutant, plant inoculation experiments were performed in growth pouches and growth chambers on the host legumes $M$. truncatula and alfalfa, using the EPS-deficient exoY mutant strain Rm7210 as a control. Five weeks after inoculation, nodulation was observed in both legumes. The seedlings inoculated with the wild-type strain Rm1021 became healthy plants with pink, elongated nodules, whereas the plants inoculated with the $c h v I$ mutant were unhealthy, with few small, round, and white nodules (Fig. 4). Even though nodules induced by the EPS-deficient exoY mutant and the $c h v I$ mutant looked similar (small, round, white nonfixing nodules), the chvI mutant induced fewer nodules (approximately 15.2\%) than the exo $Y$ mutant, which induced a similar number of nodules as the wild type did on M. truncatula and alfalfa in growth pouches (data not shown). The nodulation properties, including the average shoot dry weight (SDW), average number of nodules, and acetylene reduction activity (ARA) elicited by each strain at 5 weeks postinoculation in growth chamber, are shown in Table 2 . Both $M$. truncatula and alfalfa plants inoculated with the chvI mutant displayed significantly lower SDW than the wild-type strain, and these plants also exhibited severely reduced levels of ARA and number of nodules (Table 2). In addition, similar to the exoY mutant, the $c h v I$ mutant-derived nodules (small, round, white nonfixing nodules) typically yielded 100- to 1,000-fold fewer bacterial colonies than nodules containing Rm1021 when they were crushed and plated on M9 + suc. agar plates (data not shown). However, the symbiotic defect of the chvI mutant was fully eliminated by the ectopic expression of chvI from pCW681 (Fig. 4 ; Table 2).

Microscopic examination was used to observe whether the chvI mutant induced root hair deformation. The root hair responses caused by the $c h v I$ mutant infection were similar to the wild-type infection at 1,2 , and 3 days after inoculation (dai). M. truncatula roots inoculated with wild-type or chvI mutant $S$. meliloti were not yet morphologically distinguishable (Fig. 5).

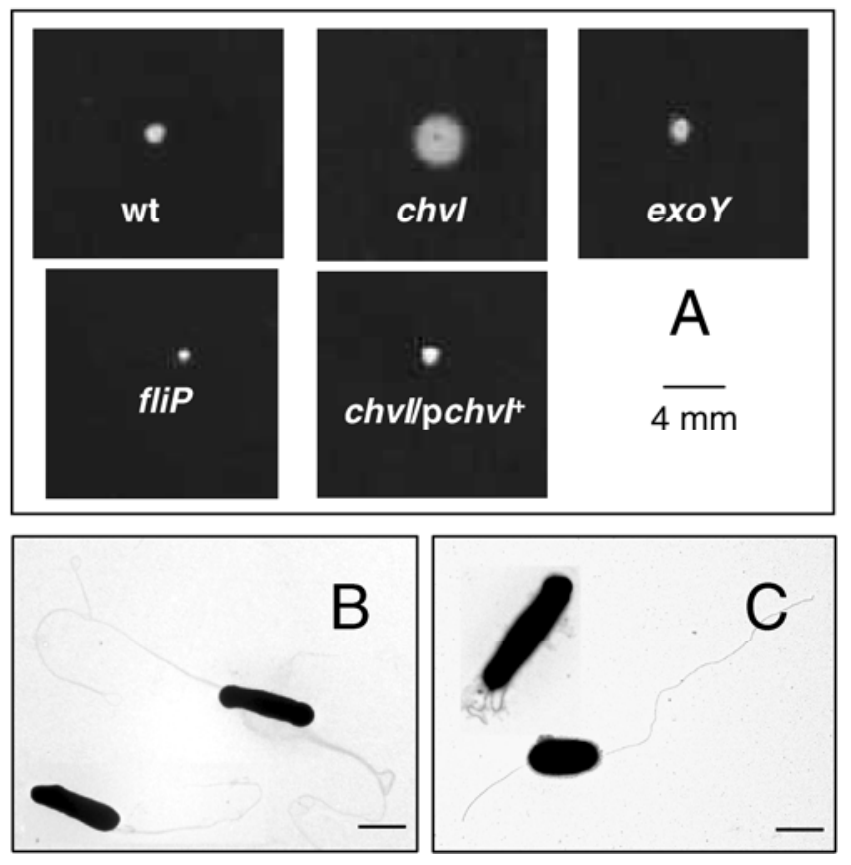

Fig. 3. Motility and flagellar status in Sinorhizobium meliloti strains. A, Motility was observed by inoculating each strain into $0.22 \%$ agar M9 + succinate $(\mathrm{M} 9+$ suc.) plates (swim plates). Relevant strain genotypes are indicated. Bacteria are shown after 4 days of growth at $30^{\circ} \mathrm{C}$. B and $\mathbf{C}$, Transmission electron microscope to examine the presence of flagella in B, Rm1021 (wild type) and C, the chvI mutant. Bacteria grown in the swim plates were negatively stained with uranyl acetate and the representative micrographs of the strains are shown at approximately $\times 10,000$ magnification. Scale bars, $1 \mu \mathrm{m}$.

Table 1. Polymer accumulation by the wild-type and mutant $\mathrm{t}^{\mathrm{a}}$

\begin{tabular}{llccc}
\hline Strain & Genotype & EPS/cell dry wt & PHB (\% cell dry wt) & Doubling time (h) \\
\hline Rm1021 & Wild type & $1.66 \pm 0.03$ & $47.1 \pm 4.46$ & $8.2 \pm 0.1$ \\
SB114 & $c h v I^{-}$ & 0 & $16.4 \pm 1.34$ & $7.7 \pm 0.2$ \\
SB1201 & $c h v I^{-}($pCW681) & $1.38 \pm 0.06$ & $39.2 \pm 2.01$ & $7.1 \pm 0.2$ \\
\hline
\end{tabular}

${ }^{a}$ Doubling time was calculated from the average for three replications, based on the standard procedure. Values are means \pm standard deviations. EPS $=$ exopolysaccharide and $\mathrm{PHB}=$ poly-3-hydroxybutyrate. 


\section{DISCUSSION}

Previously, attempts to generate complete loss-of-function mutations in either exoS or chvI in S. meliloti were unsuccessful, leading to the suggestion that both are essential genes in free-living bacteria (Osteras et al. 1995; Cheng and Walker 1998b; Wells et al. 2007). Here, it was demonstrated that it is possible to generate a loss-of-function chvI mutant in $S$. meliloti Rm1021. The mutant strain fails to grow on complex medium, exhibits lower tolerance to acidic conditions, produces significantly less PHB, and is more motile than the wildtype strain Rm1021. The profile of LPS was also altered. Further, this mutant is defective in symbiosis but induces root hair deformation as efficiently as the wild type. Together, these results indicate that ChvI is not only involved in modulating the expression of EPSI and flagella, as previously reported, but also affects regulatory networks involving the bacterial cell envelope and metabolism. Thus, the ExoS/ChvI TCS seems to have a broad role in $S$. meliloti.

Our findings demonstrate that $c h v I$ is an essential gene for growth in rich media but not an essential gene for growth in minimal media. Considerable evidence suggests that plant partners supply $\mathrm{C}_{4}$-dicarboxylic acids, such as succinate, malate, or fumarate, to nitrogen-fixing bacteroids (McDermott et al. 1989; Lodwig and Poole 2003). Because attempts to delete either exoS or $c h v I$ were unsuccessful in $S$. meliloti under standard laboratory conditions (rich media), and S. meliloti favors succinate and related dicarboxylic acids as carbon and energy sources, we decided to use the minimal medium M9+ suc. as carbon source to select for the desired mutant.

In the animal pathogen $B$. abortus, BvrS/BvrR, the ExoS/ChvI homologues, not only are critical for cell invasion and virulence (Sola-Landa et al. 1998; Lopez-Goni et al. 2002) but also affect LPS structure and outer membrane proteins (Guzman-Verri et al. 2002; Lamontagne et al. 2007). Although the role that LPS plays in the symbiosis between $S$. meliloti and alfalfa has been studied for several years, its function remains controversial and poorly understood (Clover et al. 1989; Reuhs et al. 1998). Recent studies on mutants with altered LPS demonstrated that alterations in LPS structure can affect the ability of $S$. meliloti to form an effective symbiosis (Campbell et al. 2003). The LPS profile differences between the wild-type strain Rm1021 and the chvI mutant suggest that ExoS/ChvI is essential for LPS synthesis or transport in S. meliloti.

Our data show that the chvI mutant is inhibited by acid. Previous microarray analysis reveals that the ChvG/ChvI TCS in A. tumefaciens is induced under acidic conditions (Yuan et al. 2008). The fact that gene expression is acid inducible suggests that the gene plays a role in overcoming growth inhibition under acidic conditions. Therefore, it is not surprising that a strain lacking the gene would be acid sensitive. S. meliloti requires ExoS/ChvI TCS to establish a nitrogen-fixing symbiosis with legume hosts. It remains to be determined whether $\mathrm{pH}$ plays a role in the symbiotic development process and whether ExoS/ChvI is involved in regulation of the expression of any acid-inducible genes in S. meliloti (Li et al. 2002).

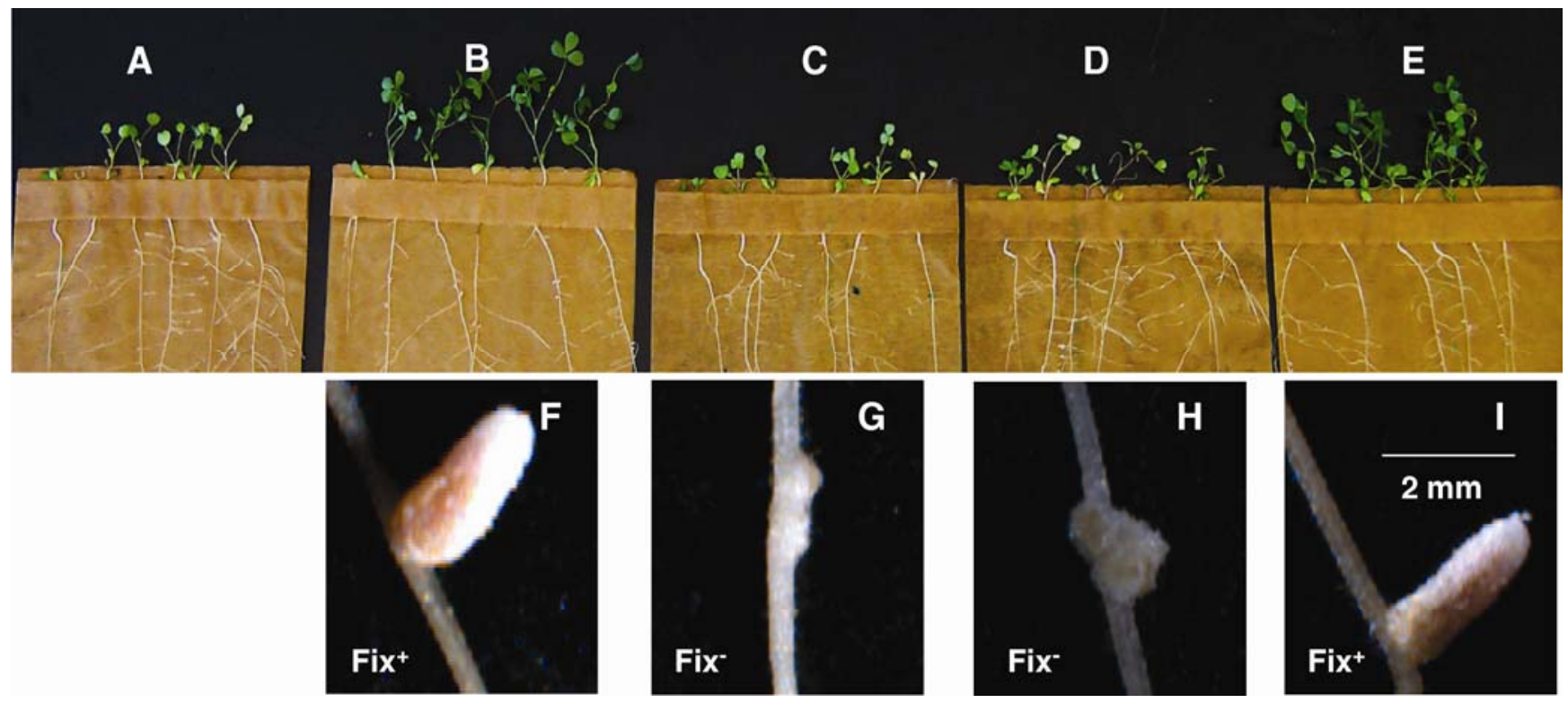

Fig. 4. Sinorhizobium meliloti chvI is required for efficient nodulation on alfalfa. Alfalfa plants were inoculated with A, no bacteria; B, wild-type strain Rm1021; C, the $c h v I$ mutant strain SB114; D, the succinoglycan-deficient exoY mutant strain Rm7210; and E, SB114 with complementing plasmid pCW681. Representative nodules were imaged 35 days after inoculation with F, Rm1021; G, SB114; H, Rm7210; and I, SB114 harboring pCW681. Symbiotic nitrogen fixation phenotypes ( $\mathrm{Fix}^{+}$or $\mathrm{Fix}^{-}$) are indicated at the bottom of each image, based on nodule color and morphology and on shoot coloration on the host plant.

Table 2. Symbiotic properties of wild-type and mutant strain on Medicago truncatula and M. sativa plants ${ }^{\mathrm{a}}$

\begin{tabular}{|c|c|c|c|c|c|c|c|}
\hline \multirow[b]{2}{*}{ Strain } & \multirow[b]{2}{*}{ Genotype } & \multicolumn{2}{|c|}{ Shoot dry weight (mg/plant) } & \multicolumn{2}{|c|}{ No. of nodules/plant } & \multicolumn{2}{|c|}{ Acetylene reduction $(\mathrm{nmol} / \mathrm{h} / \mathrm{plant})$} \\
\hline & & M. truncatula & M. sativa & M. truncatula & M. sativa & M. truncatula & M. sativa \\
\hline Rm1021 & Wild type & $10.20 \pm 0.44$ & $15.64 \pm 0.58$ & $16.96 \pm 0.32$ & $12.94 \pm 1.65$ & $13.01 \pm 1.53$ & $33.74 \pm 0.35$ \\
\hline SB114 & $\operatorname{chvI^{-}}$ & $3.75 \pm 0.25$ & $1.85 \pm 0.25$ & $0.15 \pm 0.25$ & $0.26 \pm 0.10$ & $0.37 \pm 0.12$ & $0.57 \pm 0.20$ \\
\hline SB1201 & chvI $I^{-}(\mathrm{pCW} 681)$ & $9.49 \pm 1.02$ & $10.87 \pm 0.49$ & $16.32 \pm 0.93$ & $12.08 \pm 0.52$ & $10.56 \pm 1.33$ & $30.35 \pm 0.59$ \\
\hline $\operatorname{Rm} 7210$ & exoY210::Tn5 & $2.38 \pm 0.21$ & $2.13 \pm 0.13$ & $15.33 \pm 1.97$ & $13.33 \pm 1.69$ & $0.72 \pm 0.42$ & $0.36 \pm 0.06$ \\
\hline
\end{tabular}

${ }^{a}$ Plants were grown and inoculated under growth chamber conditions as described previously (Wang et al. 2007). Dry weights, number of nodules, and acetylene reduction were measured 35 days after inoculation. Twenty-seven plants were used for each strain. Values present the means \pm standard deviations. 
Flagellar motility is a highly regulated bacterial behavior that is responsive to nutritional availability, population density, and a variety of environmental stimuli (Scharf and Schmitt 2002; Soutourina and Bertin 2003). In S. meliloti, EPS production and motility are contrarily regulated (Reed et al. 1991; Yao et al. 2004; Barnett and Fisher 2006; Wells et al. 2007; Hoang et al. 2008). The exoS96::Tn5 mutant strain, overproducing EPSI by constitutively activated ExoS protein, causes a marked reduction in the biosynthesis of flagella (Barnett et al. 2004) and consequent loss of ability of the cells to swarm and swim. EPSI overproduction is probably not the cause of the suppression of flagellum biosynthesis in the exoS96 mutant because an exoY/exoS96 double mutation is nonmotile (Yao et al. 2004). Recent work by Hoang and associates (2008) shows that the ExoS-dependent TCS suppresses motility gene expression through VisN and Rem in parallel to quorum sensing (Hoang et al. 2008). We have found that chvI mutant cells are more motile than the wild-type Rm1021 cells on swim plates. Also, both wild-type strain Rm1021 and the EPS-deficient exoY mutant Rm7210 formed similar types of colonies on the swim plates. This result demonstrates that the larger colony morphology of the $c h v I$ mutant on the swim plates is not due to the EPS phenotype. Furthermore, we confirm that cells of both wild-type Rm1021 and the chvI mutant grown on the swim plates containing minimal media (M9 + suc.) have flagella. Based on these data, we propose the following hypothesis: for ExoS/ChvI in S. meliloti, ExoS activates ChvI, ChvI controls motility genes directly or through other regulators, and the exoS96 mutation results in active ChvI and nonmotile cells lacking flagella, as previously reported (Yao et al. 2004); therefore, it is expected that a deletion of $c h v I$ exhibits greater motility than the wild type. It is still possible, however, that ExoS/ChvI may play some role in regulating chemotaxis in addition to regulating flagellum biosynthesis and EPSI biosynthesis. It should be made clear here that the Rm1021 strain used in our work has an insertion mutation in the $\exp R$ gene. This prevents the formation of EPSII (Pellock et al. 2002).

$\mathrm{PHB}$ is a major carbon storage compound in S. meliloti. Mutant analysis has demonstrated that PHB metabolism plays a role in Rhizobium-legume symbiosis (Willis and Walker 1998; Lodwig and Poole 2003; Trainer and Charles 2006; Wang et al. 2007). It has been hypothesized that PHB accumulated by the
1 dai

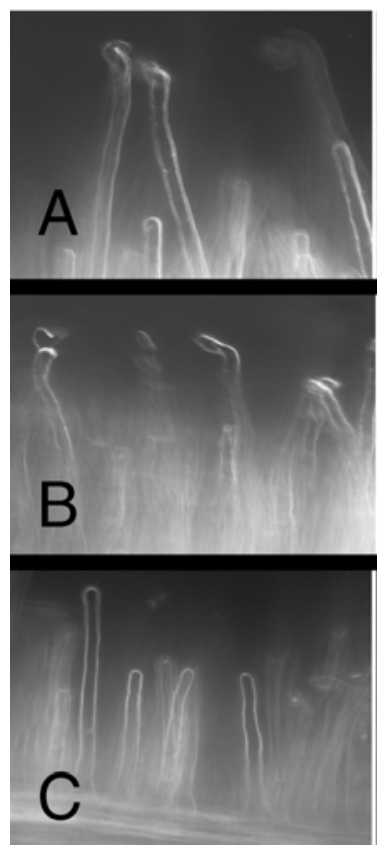

2 dai

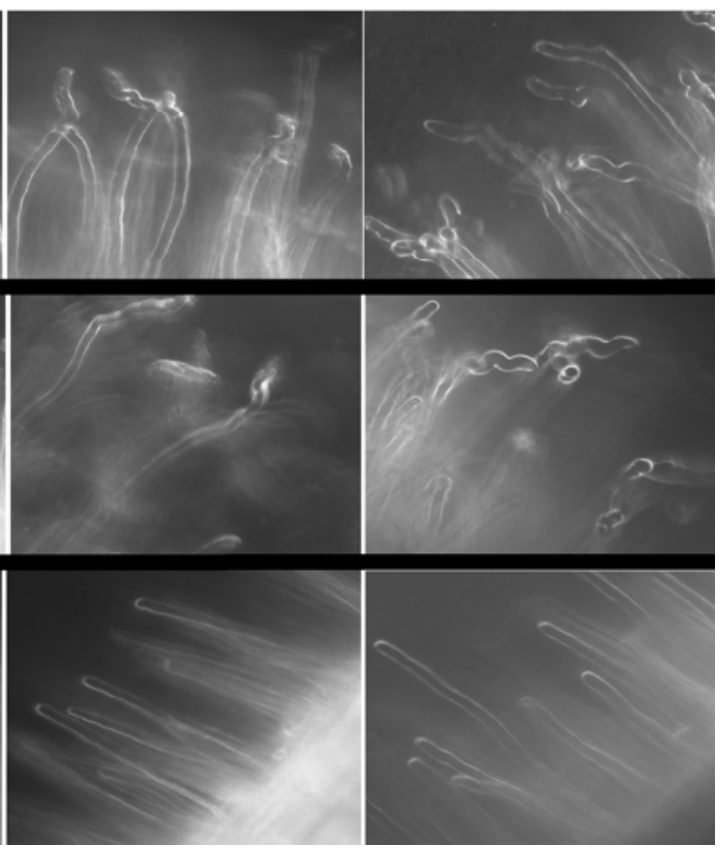

Fig. 5. Root hair deformation. Medicago truncatula roots at 1, 2, and 3 days after inoculation (dai) with Sinorhizobium meliloti A, wild-type Rm1021; B, chvI mutant; or C, uninoculated control. Magnification $\times 480$. All photos were taken of root hairs in the growing root hair zone (Wood and Newcomb 1988 ).

Table 3. Bacterial strains and plasmids

\begin{tabular}{|c|c|c|}
\hline Strain or plasmid & Genotype or relevant characteristics ${ }^{a}$ & Source or reference \\
\hline \multicolumn{3}{|l|}{ Sinorhizobium meliloti } \\
\hline Rm1021 & Derived from wild-type SU47, $\mathrm{Sm}^{\mathrm{r}}$ & Meade et al. 1982 \\
\hline Rm7210 RmG910 & exoY210::Tn5, $\mathrm{Sm}^{\mathrm{r}}, \mathrm{Nm}^{\mathrm{r}} 1021:: f l i P \Omega \mathrm{Km}$ & Leigh et al. 1985; Finan et al. 1995 \\
\hline SB114 & Rm1021 chvI-precise-deletion & This study \\
\hline SB1201 & SB114 (pCW681) & This study \\
\hline \multicolumn{3}{|l|}{ Escherichia coli } \\
\hline DH5 $\alpha$ & $\mathrm{F}^{-}$endA1 hsdR17 $\left(\mathrm{rk}^{-}, \mathrm{mk}^{+}\right)$supE44 thi-1recA1 gyrA96 relA1D(argF-lacZYA)U169 & \\
\hline & $\Phi 80 \mathrm{~d} l a c Z \Delta \mathrm{M} 15, \lambda$ & Hanahan 1983 \\
\hline DH5 $\alpha(\mathrm{pRK} 600)$ & Mobilizing strain, $\mathrm{Cm}^{\mathrm{r}}$ & Finan et al. 1986 \\
\hline \multicolumn{3}{|l|}{ Plasmids } \\
\hline pGEM-T Easy & $\mathrm{Am}^{\mathrm{r}}$, for cloning polymerase chain reaction products & Promega \\
\hline pK19mobsacB & Suicide vector, $\mathrm{Nm} / \mathrm{Km}^{\mathrm{r}}$ & Schafer et al. 1994 \\
\hline pJK1 & $c h v I$ deletion in $E c o \mathrm{R} 1$ site of $\mathrm{pK} 19 \mathrm{mobsac}, \mathrm{Km}^{\mathrm{r}}$ & This study \\
\hline pBBR1MCS-5 & Cloning vector, $\mathrm{Gm}^{\mathrm{r}}$ & Kovach et al. 1995 \\
\hline pCW681 & pBBR1MCS -5 containing the $c h v I$ gene, $\mathrm{Gm}^{\mathrm{r}}$ & This study \\
\hline
\end{tabular}

${ }^{\text {a }} \mathrm{Sm}^{\mathrm{r}}, \mathrm{Nm}^{\mathrm{r}}, \mathrm{Cm}^{\mathrm{r}}, \mathrm{Km}^{\mathrm{r}}$, and $\mathrm{Gm}^{\mathrm{r}}=$ resistant to streptomycin, neomycin, chloramphenicol, kanamycin, and gentamicin, respectively. 
bacteria prior to infection serves to fuel infection or bacteroid differentiation (Lodwig et al. 2005). A correlation between PHB metabolism and EPS synthesis has been demonstrated (Aneja et al. 2004). However, the mechanisms of regulation of PHB metabolism have not been well characterized. Here, we show that PHB production is dramatically reduced in the $c h v I$ mutant (Table 1). This raises the possibility that ExoS/ChvI may play a key role in regulating storage polymer biosynthesis and carbon metabolism in S. meliloti.

The lack of EPSI biosynthesis by the $c h v I$ mutant strain could explain the deficient symbiotic phenotype observed but, interestingly, our results clearly show that the quantity of nodules obtained was very different from the exoY mutant, deficient in EPSI biosynthesis: the exoY mutant induced significantly more nodules than the chvI mutant on M. truncatula and alfalfa after 5 weeks of inoculation. Also, we showed that there are no morphological differences yet apparent at 1,2 , and 3 dai between $M$. truncatula roots inoculated with the wild type or the chvI mutant (Fig. 5). This observation is very similar to the succinoglycan-deficient $S$. meliloti exoY mutant, which produces Nod factor in a functional form as efficiently the wild type (Leigh et al. 1985; Klein et al. 1988; Glazebrook and Walker 1989; Jones et al. 2008) and induces root hair curling as efficiently as the wild type (Cheng and Walker 1998a). This result suggests that the $c h v I$ mutant might have Nod factor gene expression as well as morphological responses similar to the wild-type strain at the early inoculation stage. Put together, the data presented in this work provide evidence that ExoS/ChvI controls a significantly broader set of phenotypes than previously reported. We are pursuing further studies using the loss-of-function $c h v I$ mutant to identify new functions controlled by the ExoS/ChvI system in S. meliloti.

\section{MATERIALS AND METHODS}

\section{Bacterial strains and general growth conditions.}

The strains and plasmids used in this work are shown in Table 3. All S. meliloti strains in this work are derivatives of $\mathrm{Rm} 1021$ (wild type) and were grown at $30^{\circ} \mathrm{C}$ in $\mathrm{TY}$ (Beringer 1974), YMB (Tombolini et al. 1995) or M9 minimal medium with carbon sources at $15 \mathrm{mM}$ as previously described (Charles and Finan 1991). Unless otherwise indicated, acid conditions refer to $\mathrm{pH} 5.5$, whereas neutral conditions refer to $\mathrm{pH}$ 7.0. Escherichia coli strains were grown at $37^{\circ} \mathrm{C}$ in LuriaBertani medium (Miller 1972). Antibiotics were used at the following concentrations: ampicillin (Am), $100 \mu \mathrm{g} / \mathrm{ml}$; kanamycin $(\mathrm{Km}), 20 \mu \mathrm{g} / \mathrm{ml}$; chloramphenicol $(\mathrm{Cm}), 25 \mu \mathrm{g} / \mathrm{ml}$; neomycin $(\mathrm{Nm}), 200 \mu \mathrm{g} / \mathrm{ml}$; tetracycline (Tc), $10 \mu \mathrm{g} / \mathrm{ml}$; gentamicin (Gm), $20 \mu \mathrm{g} / \mathrm{ml}$ (for S. meliloti) or $10 \mu \mathrm{g} / \mathrm{ml}$ (for $E$. coli); and streptomycin (Sm), $200 \mu \mathrm{g} / \mathrm{ml}$. Media were solidified by the addition of $1.5 \%$ (wt/vol) agar. Liquid cultures were shaken at $220 \mathrm{rpm}$.

\section{Construction of the $\operatorname{chvI}$ deletion mutant.}

The loss-of-function $c h v I$ mutant was constructed by crossover PCR (Sukdeo and Charles 2003) using the suicide vector pK19mobsacB (Schafer et al. 1994), which cannot replicate in S. meliloti. In brief, PCR on Rm1021 genomic DNA template was used to generate fragments on either side of the chvI sequence targeted for deletion. For amplification of the N-terminal end of $c h v I$, primers A (5' TGAATTTCGTCGGAACAT ACC $\left.3^{\prime}\right)$ and B (5' GCCGTCGACGAGCGAGAGGCACTTAT TCTACTCCGTTTCGA $3^{\prime}$ ) were used, resulting in an amplification product of $329 \mathrm{bp}$. For amplification of the C-terminal end of $c h v I$, primers C (5' TGCCTCTCGCTCGTCGACGGC GGGAGCGAGCCGGCGGATTG 3') and D (5' GATTCGAC GTTCGCTACTGC $3^{\prime}$ ) were used, resulting in an amplification product of $385 \mathrm{bp}$. Primers B and C contained 21-bp complementary sequences at their $5^{\prime}$ ends, as underlined. The PCR products resulting from these two amplifications were gel purified and combined as templates for a PCR reaction using primer pairs A and D. A fragment of the desired 693-bp size was obtained and ligated into the pGEM-T Easy Vector (Promega Corp., Madison, WI, U.S.A.), and the sequence was confirmed to be correct; the fragment was then subcloned into pK19mobsacB to produce plasmid pJK1. Gene replacement in Rm1021 was carried out by first introducing pJK1 by triparental mating, selecting for Sm- and Nm-resistant single-crossover cointegrants. Subsequent selection for double-crossover events was carried out on M9 + suc. agar containing 5\% sucrose, followed by screening for $\mathrm{Nm}$ sensitivity. The precise deletion of $c h v I$ was confirmed by PCR amplification using flanking primers and DNA sequencing. The corresponding strain was designated SB114.

In order to complement the $S$. meliloti chvI mutant, a 1,036bp fragment containing $c h v I$ under the control of its own promoter was amplified by PCR using primers chvI-F09 (5' TTTAAGCTTGGATCAGGTGCTTTTGCACT $3^{\prime}$ ) and chvIR09 (5' TTTGGATCCAAAACTGTGTGCGGTTTTCC $\left.3^{\prime}\right)$ (introduced restriction sites underlined) and Rm1021 genomic DNA as a template. The fragment was digested with HindIII and BamHI and ligated into a HindIII-BamHI-digested pBBR1MCS-5 plasmid (Kovach et al. 1995), and the resulting plasmid was named pCW681. The plasmid pCW681 was transformed into $E$. coli $\mathrm{DH} 5 \alpha$ competent cells. The clone was confirmed by PCR and DNA sequencing prior to introduction into $S$. meliloti chvI mutant by triparental mating using helper plasmid pRK600 (Finan et al. 1986).

\section{Motility tests and TEM.}

Motility was assessed by using swim agar plates as described (Wells et al. 2007) but using M9 + suc. medium instead of rich medium. Briefly, colonies were taken from the surface of regular $1.5 \%$ agar plates with $10-\mu$ l pipette tips, and inserted into $0.22 \%$ agar plates. Plates were subsequently dried for approximately $2 \mathrm{~h}$ and then transferred to a $30^{\circ} \mathrm{C}$ incubator, where they were incubated upside down. Motility was scored after 4 days.

For electron microscopy analysis, bacterial cells were picked from the swim agar plates, fixed by adding $2 \%$ formaldehyde, washed with phosphate buffered saline, and applied to carbon-coated copper grids. Samples were washed twice with distilled water, negatively stained for $30 \mathrm{~s}$ in $0.5 \%$ uranyl acetate, and then rinsed three times $(10 \mathrm{~s}$ each) by submersion in aliquots of Milli-Q water. Imaging was performed with a Zeiss 10CA Transmission Electron Microscope (Carl Zeiss Inc., Oberkochen, Germany) at $60 \mathrm{kV}$ of accelerating voltage at room temperature.

\section{LPS isolation and analysis.}

The procedure for preparation and visualization of $S$. meliloti LPS was similar to that employed by Reuhs and associates (1993) with the following modifications: cells were grown to late log phase in M9 + suc. medium, after which 1.5 $\mathrm{ml}$ of each sample was pelleted by centrifugation for $5 \mathrm{~min}$ at $13,000 \times g$. The pellets were washed twice with water before being resuspended in $50 \mu \mathrm{l}$ of $65^{\circ} \mathrm{C}$ water. To the cell suspension, $50 \mu \mathrm{l}$ of $65^{\circ} \mathrm{C}$ phenol was added, followed by vortexing, incubation at $65^{\circ} \mathrm{C}$ for $15 \mathrm{~min}$, and cooling on ice for $10 \mathrm{~min}$. The samples were then centrifuged at $13,000 \times g$ for $10 \mathrm{~min}$, the water phase was set aside, and the phenol phase was heated to $65^{\circ} \mathrm{C}$. Then, $50 \mu \mathrm{l}$ of $65^{\circ} \mathrm{C}$ water was added to the hot phenol phase and the above procedure was repeated. The water phases (containing LPS) were combined and mixed with sam- 
ple buffer (62 mM Tris $\mathrm{HCl}$ [pH 6.8], 2\% sodium dodecyl sulfate [SDS], $5 \% \beta$-mercaptoethanol, $10 \%$ glycerol, and $0.03 \%$ bromophenol blue) in a 5:1 ratio.

A volume of $5 \mu \mathrm{l}$ of the final preparation was then applied to a standard SDS acrylamide minigel (Laemmli 1970) containing $15 \%$ acrylamide and visualized by silver staining (Hitchcock and Brown 1983).

\section{Quantification of EPS and PHB.}

The EPS content was determined as previously described (Marroqui et al. 2001) from cultures grown in YMB for 4 days at $30^{\circ} \mathrm{C}$. The concentration of PHB was determined from cells grown in YMB medium using the spectrophotometric technique (Law and Slepecky 1961) as modified by Peoples and Sinskey (1989).

\section{Root hair deformation phenotype assay.}

Root hair responses by $S$. meliloti were analyzed on microscope slides as described by Gage and associates (1996) with the following modifications. $M$. truncatula Jemalong (line A17) seed were sterilized and germinated as described previously (Wang et al. 2007). Two seedlings, 1 to $2 \mathrm{~cm}$ long, were placed on top of the microscope slides which had been covered with $2 \mathrm{ml}$ of BNM medium (Ehrhardt et al. 1992) in 1.1\% agarose. The seedlings were covered with a single layer of washed dialysis membrane (12,000 to 14,000 molecular weight cutoff) after being inoculated with $0.1 \mathrm{ml}$ of a 1:100 dilution in sterile water of $S$. meliloti cells grown to saturation in $\mathrm{M} 9+$ suc. medium and washed once in sterilized water. The slides were then placed in a $250-\mathrm{ml}$ beaker containing $30 \mathrm{ml}$ of liquid BNM medium and covered loosely with Saran Wrap, and the beaker was placed in a growth chamber $\left(26^{\circ} \mathrm{C}\right.$, light and dark periods of 16 and $8 \mathrm{~h}$, respectively). Root hairs were examined and photographs were taken as described by Wood and Newcomb (1988) using a Zeiss Axioskop Microscope.

\section{Symbiotic assays.}

Symbiotic phenotype assays were performed with $M$. truncatula Jemalong (line A17) and alfalfa ( $M$. sativa cv. Iroquois) plants in growth pouches and growth chambers as described previously (Wang et al. 2007). Shoot dry weights, number of nodules, and nitrogen fixation activities (acetylene reduction) were determined 5 weeks after inoculation. Stained nodules were observed and imaged through a $\times 0.8$ objective lens on a Leica Microscope.

\section{Statistical analysis.}

All the data presented for PHB, EPS, nodule numbers, acetylene reduction assays, and plant dry weight are presented as means with standard errors. The significance of the results was assessed using Student's $t$ test.

\section{ACKNOWLEDGMENTS}

This work was supported by the Virginia Bioinformatics Institute. Work in the lab of T. C. Charles was supported by NSERC. We thank B. Glick for critical reading of the manuscript, V. Shulaev and J. Shuman for technical assistance with the gas chromatography, A. Pereira and M. Ambavaram for the use of their microscope, K. Lowe for help with TEM, T. Finan for providing the 1021::fliPSKm mutant RmG910, and H. P. Cheng for providing the exoY210::Tn5 mutant strain $\mathrm{Rm} 7210$.

\section{LITERATURE CITED}

Aneja, P., Dai, M., Lacorre, D. A., Pillon, B., and Charles, T. C. 2004. Heterologous complementation of the exopolysaccharide synthesis and carbon utilization phenotypes of Sinorhizobium meliloti Rm1021 polyhydroxyalkanoate synthesis mutants. FEMS (Fed. Mol. Microbiol. Soc.) Microbiol. Lett. 239:277-283.
Azcarate-Peril, M. A., McAuliffe, O., Altermann, E., Lick, S., Russell, W. M., and Klaenhammer, T. R. 2005. Microarray analysis of a two-component regulatory system involved in acid resistance and proteolytic activity in Lactobacillus acidophilus. Appl. Environ. Microbiol. 71:5794-5804.

Barnett, M. J., and Fisher, R. F. 2006. Global gene expression in the rhizobial-legume symbiosis. Symbiosis 42:1-24.

Barnett, M. J., Toman, C. J., Fisher, R. F., and Long, S. R. 2004. A dualgenome Symbiosis Chip for coordinate study of signal exchange and development in a prokaryote-host interaction. Proc. Natl. Acad. Sci. U.S.A. 101:16636-16641.

Beringer, J. E. 1974. R factor transfer in Rhizobium leguminosarum. J. Gen. Microbiol. 84:188-198.

Brewin, N. J. 1991. Development of the legume root nodule. Annu. Rev. Cell Biol. 7:191-226.

Campbell, G. R., Sharypova, L. A., Scheidle, H., Jones, K. M., Niehaus, K., Becker, A., and Walker, G. C. 2003. Striking complexity of lipopolysaccharide defects in a collection of Sinorhizobium meliloti mutants. J. Bacteriol. 185:3853-3862.

Charles, T. C., and Finan, T. M. 1991. Analysis of a 1600-kilobase Rhizobium meliloti megaplasmid using defined deletions generated in vivo. Genetics 127:5-20.

Charles, T. C., and Nester, E. W. 1993. A chromosomally encoded twocomponent sensory transduction system is required for virulence of Agrobacterium tumefaciens. J. Bacteriol. 175:6614-6625.

Cheng, H. P., and Walker, G. C. 1998a. Succinoglycan is required for initiation and elongation of infection threads during nodulation of alfalfa by Rhizobium meliloti. J. Bacteriol. 180:5183-5191.

Cheng, H. P., and Walker, G. C. 1998b. Succinoglycan production by Rhizobium meliloti is regulated through the ExoS-ChvI two-component regulatory system. J. Bacteriol. 180:20-26.

Cheng, H. P., and Yao, S. Y. 2004. The key Sinorhizobium meliloti succinoglycan biosynthesis gene exoY is expressed from two promoters FEMS (Fed. Mol. Microbiol. Soc.) Microbiol. Lett. 231:131-136.

Clover, R. H., Kieber, J., and Signer, E. R. 1989. Lipopolysaccharide mutants of Rhizobium meliloti are not defective in symbiosis. J. Bacteriol. 171:3961-3967.

Doherty, D., Leigh, J. A., Glazebrook, J., and Walker, G. C. 1988. Rhizobium meliloti mutants that overproduce the $R$. meliloti acidic calcofluorbinding exopolysaccharide. J. Bacteriol. 170:4249-4256.

Ehrhardt, D. W., Atkinson, E. M., and Long, S. R. 1992. Depolarization of alfalfa root hair membrane potential by Rhizobium meliloti Nod factors. Science 256:998-1000.

Finan, T. M., Kunkel, B., DeVos, G. F., and Signer, E. R. 1986. Second symbiotic megaplasmid in Rhizobium meliloti carrying exopolysaccharide and thiamine synthesis genes. J. Bacteriol. 167:66-72.

Finan, T. M., Gough, C., and Trucher, G. 1995. Similarity between the Rhizobium meliloti fliP gene and pathogenicity-associated genes from animal and plant pathogens. Gene 152:65-77.

Gage, D. J., Bobo, T., and Long, S. R. 1996. Use of green fluorescent protein to visualize the early events of symbiosis between Rhizobium meliloti and alfalfa (Medicago sativa). J. Bacteriol. 178:7159-7166.

Glazebrook, J., and Walker, G. C. 1989. A novel exopolysaccharide can function in place of the calcofluor-binding exopolysaccharide in nodulation of alfalfa by Rhizobium meliloti. Cell 56:661-672.

Guzman-Verri, C., Manterola, L., Sola-Landa, A., Parra, A., Cloeckaert, A., Garin, J., Gorvel, J. P., Moriyon, I., Moreno, E., and Lopez-Goni, I. 2002. The two-component system BvrR/BvrS essential for Brucella abortus virulence regulates the expression of outer membrane proteins with counterparts in members of the Rhizobiaceae. Proc. Natl. Acad. Sci. U.S.A. 99:12375-12380.

Hanahan, D. 1983. Studies on transformation of Escherichia coli with plasmids. J. Mol. Biol. 166:557-580.

Hitchcock, P. J., and Brown, T. M. 1983. Morphological heterogeneity among Salmonella lipopolysaccharide chemotypes in silver-stained polyacrylamide gels. J. Bacteriol. 154:269-277.

Hoang, H. H., Gurich, N., and Gonzalez, J. E. 2008. Regulation of motility by the ExpR/Sin quorum-sensing system in Sinorhizobium meliloti. J. Bacteriol. 190:861-871.

Jones, K. M., Kobayashi, H., Davies, B. W., Taga, M. E., and Walker, G. C. 2007. How rhizobial symbionts invade plants: The SinorhizobiumMedicago model. Nat. Rev. Microbiol. 5:619-633.

Jones, K. M., Sharopova, N., Lohar, D. P., Zhang, J. Q., VandenBosch, K. A., and Walker, G. C. 2008. Differential response of the plant Medicago truncatula to its symbiont Sinorhizobium meliloti or an exopolysaccharide-deficient mutant. Proc. Natl. Acad. Sci. U.S.A. 105:704-709.

Klein, S., Hirsch, A. M., Smith, C. A., and Signer, E. R. 1988. Interaction of nod and exo Rhizobium meliloti in alfalfa nodulation. Mol. Plant-Microbe Interact. 1:94-100.

Kovach, M. E., Elzer, P. H., Hill, D. S., Robertson, G. T., Farris, M. A., Roop, R. M., 2nd, and Peterson, K. M. 1995. Four new derivatives of 
the broad-host-range cloning vector pBBR1MCS, carrying different antibiotic-resistance cassettes. Gene 166:175-176.

Laemmli, U. K. 1970. Cleavage of structural proteins during the assembly of the head of bacteriophage T4. Nature 227:680-685.

Lamontagne, J., Butler, H., Chaves-Olarte, E., Hunter, J., Schirm, M., Paquet, C., Tian, M., Kearney, P., Hamaidi, L., Chelsky, D., Moriyon, I., Moreno, E., and Paramithiotis, E. 2007. Extensive cell envelope modulation is associated with virulence in Brucella abortus. J. Proteome Res. 6:1519-1529.

Law, J. H., and Slepecky, R. A. 1961. Assay of poly- $\beta$-hydroxybutyric acid. J. Bacteriol. 82:33-36.

Leigh, J. A., Signer, E. R., and Walker, G. C. 1985. Exopolysaccharide-deficient mutants of Rhizobium meliloti that form ineffective nodules. Proc. Natl. Acad. Sci. U.S.A. 82:6231-6235.

Li, L., Jia, Y., Hou, Q., Charles, T. C., Nester, E. W., and Pan, S. Q. 2002. A global pH sensor: Agrobacterium sensor protein ChvG regulates acidinducible genes on its two chromosomes and Ti plasmid. Proc. Natl. Acad. Sci. U.S.A. 99:12369-12374.

Lodwig, E. M., and Poole, P. S. 2003. Metabolism of rhizobium bacteroids. Crit. Rev. Plant Sci. 22:37-78.

Lodwig, E. M., Leonard, M., Marroqui, S., Wheeler, T. R., Findlay, K., Downie, J. A., and Poole, P. S. 2005. Role of polyhydroxybutyrate and glycogen as carbon storage compounds in pea and bean bacteroids. Mol. Plant-Microbe Interact. 18:67-74.

Long, S. R. 1989. Rhizobium-legume nodulation: Life together in the underground. Cell 56:203-214.

Long, S. R. 2001. Genes and signals in the Rhizobium-legume symbiosis. Plant Physiol. 125:69-72.

Lopez-Goni, I., Guzman-Verri, C., Manterola, L., Sola-Landa, A., Moriyon, I., and Moreno, E. 2002. Regulation of Brucella virulence by the twocomponent system BvrR/BvrS. Vet. Microbiol. 90:329-339.

Manterola, L., Moriyon, I., Moreno, E., Sola-Landa, A., Weiss, D. S., Koch, M. H., Howe, J., Brandenburg, K., and Lopez-Goni, I. 2005. The lipopolysaccharide of Brucella abortus BvrS/BvrR mutants contains lipid A modifications and has higher affinity for bactericidal cationic peptides. J. Bacteriol. 187:5631-5639.

Marroqui, S., Zorreguieta, A., Santamaria, C., Temprano, F., Soberon, M. Megias, M., and Downie, J. A. 2001. Enhanced symbiotic performance by Rhizobium tropici glycogen synthase mutants. J. Bacteriol. 183:854-864.

McDermott, T. R., Griffith, S. M., Vance, C. P., and Graham, P. H. 1989. Carbon metabolism in Bradyrhizobium japonicum bacteroids. FEMS (Fed. Mol. Microbiol. Soc.) Microbiol. Rev. 63:327-340.

Meade, H. M., Long, S. R., Ruvkun, G. B., Brown, S. E., and Ausubel, F M. 1982. Physical and genetic characterization of symbiotic and auxotrophic mutants of Rhizobium meliloti induced by transposon mutagenesis. J. Bacteriol. 149:114-122.

Mendrygal, K. E., and Gonzalez, J. E. 2000. Environmental regulation of exopolysaccharide production in Sinorhizobium meliloti. J. Bacteriol. 182:599-606

Miller, J. H. 1972. Experiments in Molecular Genetics. Cold Spring Harbor Laboratory, Cold Spring Harbor, NY, U.S.A.

Nishino, K., Honda, T., and Yamaguchi, A. 2005. Genome-wide analyses of Escherichia coli gene expression responsive to the BaeSR two-component regulatory system. J. Bacteriol. 187:1763-1772.

Oke, V., and Long, S. R. 1999. Bacteroid formation in the Rhizobium-legume symbiosis. Curr. Opin. Microbiol. 2:641-646.

Osteras, M., Stanley, J., and Finan, T. M. 1995. Identification of Rhizobiumspecific intergenic mosaic elements within an essential two-component regulatory system of Rhizobium species. J. Bacteriol. 177:5485-5494.

Pellock, B. J., Cheng, H. P., and Walker, G. C. 2000. Alfalfa root nodule invasion efficiency is dependent on Sinorhizobium meliloti polysaccharides. J. Bacteriol. 182:4310-4318.

Pellock, B. J., Teplitski, M., Boinay, R. P., Bauer, W. D., and Walker, G. C. 2002. A LuxR homolog controls production of symbiotically active extracellular polysaccharide II by Sinorhizobium meliloti. J. Bacteriol. 184:5067-5076

Peoples, O. P., and Sinskey, A. J. 1989. Poly- $\beta$-hydroxybutyrate biosynthesis in Alcaligenes eutrophus H16. Characterization of the genes encoding $\beta$-ketothiolase and acetoacetyl-CoA reductase. J. Biol. Chem. 264:15293-15297.

Perret, X., Staehelin, C., and Broughton, W. 2000. Molecular basis of symbiotic promiscuity. Microbiol. Mol. Biol. Rev. 64:180-201.

Reed, J. W., Glazebrook, J., and Walker, G. C. 1991. The exoR gene of Rhizobium meliloti affects RNA levels of other exo genes but lacks homology to known transcriptional regulators. J. Bacteriol. 173:3789-3794.
Reuhs, B. L., Carlson, R. W., and Kim, J. S. 1993. Rhizobium fredii and Rhizobium meliloti produce 3-deoxy-D-manno-2-octulosonic acid-containing polysaccharides that are structurally analogous to group II K antigens (capsular polysaccharides) found in Escherichia coli. J. Bacteriol. 175:3570-3580.

Reuhs, B. L., Geller, D. P., Kim, J. S., Fox, J. E., Kolli, V. S. K., and Pueppke, S. G. 1998. Sinorhizobium fredii and Sinorhizobium meliloti produce structurally conserved lipopolysaccharides and strain-specific K antigens. Appl. Environ. Microbiol. 64:4930-4938.

Schafer, A., Tauch, A., Jager, W., Kalinowski, J., Thierbach, G., and Puhler, A. 1994. Small mobilizable multi-purpose cloning vectors derived from the Escherichia coli plasmids pK18 and pK19: Selection of defined deletions in the chromosome of Corynebacterium glutamicum. Gene 145:69-73.

Scharf, B., and Schmitt, R. 2002. Sensory transduction to the flagellar motor of Sinorhizobium meliloti. J. Mol. Microbiol. Biotechnol. 4:183-186.

Schultze, M., and Kondorosi, A. 1998. Regulation of symbiotic root nodule development. Annu. Rev. Genet 32:33-57.

Sola-Landa, A., Pizarro-Cerda, J., Grillo, M. J., Moreno, E., Moriyon, I., Blasco, J. M., Gorvel, J. P., and Lopez-Goni, I. 1998. A two-component regulatory system playing a critical role in plant pathogens and endosymbionts is present in Brucella abortus and controls cell invasion and virulence. Mol. Microbiol. 29:125-138.

Soutourina, O. A., and Bertin, P. N. 2003. Regulation cascade of flagellar expression in Gram-negative bacteria. FEMS (Fed. Mol. Microbiol. Soc.) Microbiol. Rev. 27:505-523.

Sukdeo, N., and Charles, T. C. 2003. Application of crossover-PCR-mediated deletion-insertion mutagenesis to analysis of the $b d h A-x d h A 2$ $x d h B 2$ mixed-function operon of Sinorhizobium meliloti. Arch. Microbiol. 179:301-304

Tombolini, R., Povolo, S., Buson, A., Squartini, A., and Nuti, M. P. 1995. Poly- $\beta$-hydroxybutyrate (PHB) biosynthetic genes in Rhizobium meliloti 41. Microbiology 141:2553-2559.

Trainer, M. A., and Charles, T. C. 2006. The role of PHB metabolism in the symbiosis of rhizobia with legumes. Appl. Microbiol. Biotechnol. 71:377-386.

Tsolis, R. M. 2002. Comparative genome analysis of the alpha-proteobacteria: Relationships between plant and animal pathogens and host specificity. Proc. Natl. Acad. Sci. U.S.A. 99:12503-12505.

Ulrich, L. E., Koonin, E. V., and Zhulin, I. B. 2005. One-component systems dominate signal transduction in prokaryotes. Trends Microbiol. 13:52-56.

van Rhijn, P., and Vanderleyden, J. 1995. The Rhizobium-plant sybmbiosis. Microbiol. Rev. 59:124-142.

Wang, C., Saldanha, M., Sheng, X., Shelswell, K. J., Walsh, K. T., Sobral, B. W. S., and Charles, T. C. 2007. Roles of poly-3-hydroxybutyrate (PHB) and glycogen in symbiosis of Sinorhizobium meliloti with Medicago sp. Microbiology 153:388-398.

Wells, D. H., and Long, S. R. 2002. The Sinorhizobium meliloti stringent response affects multiple aspects of symbiosis. Mol. Microbiol. 43:1115-1127.

Wells, D. H., Chen, E. J., Fisher, R. F., and Long, S. R. 2007. ExoR is genetically coupled to the ExoS-ChvI two-component system and located in the periplasm of Sinorhizobium meliloti. Mol. Microbiol. 64:647-664.

Willis, L. B., and Walker, G. C. 1998. The $p h b C$ (poly- $\beta$-hydroxybutyrate synthase) gene of Rhizobium (Sinorhizobium) meliloti and characterization of phbC mutants. Can. J. Microbiol. 44:554-564.

Wood, S. M., and Newcomb, W. 1988. Nodule morphogenesis: The early infection of alfalfa (Medicago sativa) root hairs by Rhizobium meliloti. Can. J. Bot. 67:3108-3122.

Yao, S. Y., Luo, L., Har, K. J., Becker, A., Ruberg, S., Yu, G. Q., Zhu, J. B. and Cheng, H. P. 2004. Sinorhizobium meliloti ExoR and ExoS proteins regulate both succinoglycan and flagellum production. J. Bacteriol. 186:6042-6049.

Yuan, Z. C., Liu, P., Saenkham, P., Kerr, K., and Nester, E. W. 2008. Transcriptome profiling and functional analysis of Agrobacterium tumefaciens reveals a general conserved response to acidic conditions ( $\mathrm{pH} 5.5)$ and a complex acid-mediated signaling involved in Agrobacteriumplant interactions. J. Bacteriol. 190:494-507.

\section{AUTHOR-RECOMMENDED INTERNET RESOURCE}

RhizoBase website: genome.kazusa.or.jp/rhizobase 